\title{
\begin{tabular}{l|l} 
Mitraries & DSpace@MIT
\end{tabular}
}

\author{
MIT Open Access Articles
}

\section{Lower hybrid wave edge power loss quantification on the Alcator C-Mod tokamak}

The MIT Faculty has made this article openly available. Please share how this access benefits you. Your story matters.

Citation: Faust, I. C., D. Brunner, B. LaBombard, R. R. Parker, J. L. Terry, D. G. Whyte, S. G. Baek, et al. "Lower Hybrid Wave Edge Power Loss Quantification on the Alcator C-Mod Tokamak." Physics of Plasmas 23, no. 5 (May 2016): 056115.

As Published: http://dx.doi.org/10.1063/1.4951736

Publisher: American Institute of Physics (AIP)

Persistent URL: http://hdl.handle.net/1721.1/108781

Version: Author's final manuscript: final author's manuscript post peer review, without publisher's formatting or copy editing

Terms of use: Creative Commons Attribution-Noncommercial-Share Alike 


\section{Lower hybrid wave edge power loss quantification on the Alcator C-Mod tokamak}

I. C. Faust', D. Brunner, B. LaBombard, R. R. Parker, J. L. Terry, D. G. Whyte, S. G. Baek, E. Edlund, A. E. Hubbard, J.'W. Hughes, A. Q. Kuang, M. L. Reinke, S. Shiraiwa, G. M. Wallace, and J. R. Walk

Citation: Physics of Plasmas 23, 056115 (2016); doi: 10.1063/1.4951736

View online: http://dx.doi.org/10.1063/1.4951736

View Table of Contents: http://aip.scitation.org/toc/php/23/5

Published by the American Institute of Physics

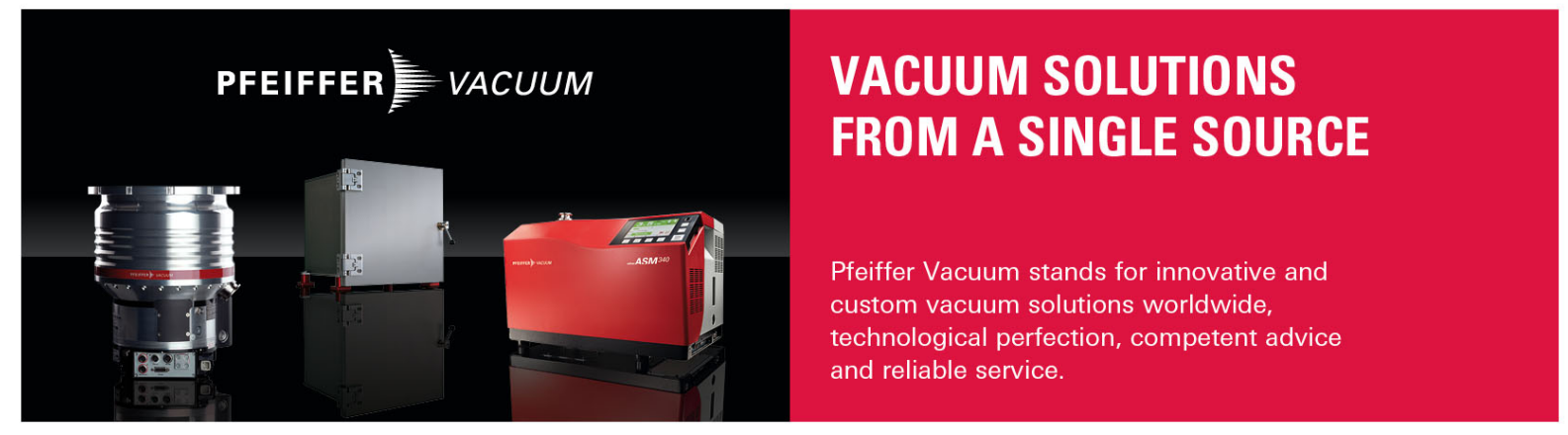




\section{Lower hybrid wave edge power loss quantification on the Alcator C-Mod tokamak}

I. C. Faust ${ }^{1, \text { a) }}$ D. Brunner, ${ }^{1}$ B. LaBombard, ${ }^{1}$ R. R. Parker, ${ }^{1}$ J. L. Terry, ${ }^{1}$ D. G. Whyte,${ }^{1}$

S. G. Baek, ${ }^{1}$ E. Edlund, ${ }^{1}$ A. E. Hubbard, ${ }^{1}$ J. W. Hughes, ${ }^{1}$ A. Q. Kuang, ${ }^{1}$ M. L. Reinke,${ }^{2}$

S. Shiraiwa, ${ }^{1}$ G. M. Wallace, ${ }^{1}$ and J. R. Walk ${ }^{1}$

${ }^{1}$ MIT Plasma Science and Fusion Center, Cambridge, Massachusetts 02139, USA

${ }^{2}$ Oak Ridge National Laboratory, Oak Ridge, Tennessee 37831, USA

(Received 16 March 2016; accepted 11 May 2016; published online 20 May 2016)

For the first time, the power deposition of lower hybrid RF waves into the edge plasma of a diverted tokamak has been systematically quantified. Edge deposition represents a parasitic loss of power that can greatly impact the use and efficiency of Lower Hybrid Current Drive (LHCD) at reactor-relevant densities. Through the use of a unique set of fast time resolution edge diagnostics, including innovative fast-thermocouples, an extensive set of Langmuir probes, and a $\mathrm{Ly}_{\alpha}$ ionization camera, the toroidal, poloidal, and radial structure of the power deposition has been simultaneously determined. Power modulation was used to directly isolate the RF effects due to the prompt $\left(t<\tau_{E}\right)$ response of the scrape-off-layer (SOL) plasma to Lower Hybrid Radiofrequency (LHRF) power. LHRF power was found to absorb more strongly in the edge at higher densities. It is found that a majority of this edge-deposited power is promptly conducted to the divertor. This correlates with the loss of current drive efficiency at high density previously observed on Alcator C-Mod, and displaying characteristics that contrast with the local RF edge absorption seen on other tokamaks. Measurements of ionization in the active divertor show dramatic changes due to LHRF power, implying that divertor region can be a key for the LHRF edge power deposition physics. These observations support the existence of a loss mechanism near the edge for LHRF at high density $\left(n_{e}>1.0 \times 10^{20}\left(\mathrm{~m}^{-3}\right)\right)$. Results will be shown addressing the distribution of power within the SOL, including the toroidal symmetry and radial distribution. These characteristics are important for deducing the cause of the reduced LHCD efficiency at high density and motivate the tailoring of wave propagation to minimize SOL interaction, for example, through the use of high-field-side launch. Published by AIP Publishing. [http://dx.doi.org/10.1063/1.4951736]

\section{INTRODUCTION}

Limitations in inductive and bootstrap current generation require the use of other means in driving current for future steady-state, reactor-relevant tokamaks. The Alcator C-Mod Lower Hybrid current drive (LHCD) system generates current by launching radiofrequency waves using a phased waveguide array antenna. ${ }^{1}$ This power generates a non-Maxwellian tail of "passing" fast electron tail via Landau damping and is an efficient and effective way of sustaining the necessary toroidal current. ${ }^{2}$ On Alcator C-Mod, LHCD can sustain fully non-inductive plasmas ${ }^{3}$ and can test the viability of these systems in high density, high magnetic field, diverted plasmas. ${ }^{4}$

While this method of current generation is very efficient ${ }^{5}$ at low density, the propagation and damping of LHCD power is complicated. The absorption of Lower Hybrid (LH) waves via Landau damping requires significant upshift of the $n_{\|}$of the wave. ${ }^{6}$ On Alcator C-Mod, this upshift of the wave may require several traversals through the plasma periphery and core. This complicated propagation can lead to a host of processes which can change the nature of the lower hybrid wave absorption. ${ }^{6-9}$ Some of the changes in the wave

Note: Paper VI2 3, Bull. Am. Phys. Soc. 60, 389 (2015).

${ }^{a)}$ Invited speaker. propagation and absorption can weaken the efficacy of LHCD and limit its utility.

At line-average densities above $1 \times 10^{20}\left(\mathrm{~m}^{-3}\right)$, a significant reduction in current drive efficiency is observed on Alcator C-Mod. ${ }^{10}$ This loss is observed in diverted discharges while inner-wall-limited discharges maintain effective current drive $\mathrm{e}^{11}$ up to higher density $\left(n_{e}>1.5 \times 10^{20}\left(\mathrm{~m}^{-3}\right)\right)$. Measurements of high energy bremsstrahlung, ${ }^{12}$ related to the fast electron tail population, display an exponential decrease in emission with increasing density in diverted plasmas. This reduction in current drive efficiency signifies that the propagation and absorption of the lower hybrid waves are changing. Understanding this current drive loss is extremely important for operation and design of current drive systems on future tokamaks since densities above $1 \times 10^{20}\left(\mathrm{~m}^{-3}\right)$ are foreseen on ITER and DEMO-like devices.

Anomalous current drive loss (when the current drive efficiency is not proportional to $\bar{n}_{e}^{-1}$ ) has been observed on a number of other tokamaks, both in diverted and limited plasmas. Edge effects were blamed for reducing the current drive efficiency in nearly all cases. Limited tokamaks found parametric instabilities and density fluctuations as possible mechanisms for changing the wave in the edge. ${ }^{13-15}$ Diverted discharges have displayed measurable fast electron edge losses $(5 \%-10 \% \text { of the input power })^{16}$ or issues with 
accessibility. ${ }^{17}$ The current drive efficiency has been observed to be a function of edge parameters through the use of lithiation. ${ }^{18}$ The strong discrepancy in current drive efficiency between limited and diverted discharges on C-Mod is also consistent with the hypothesis that interaction between the wave and edge plasma is playing a significant role.

The loss of current drive and the reduction in the fast electron population in C-Mod diverted plasmas highlights the importance of the scrape-off-layer (SOL). The edge plasma in diverted discharges changes with applied LH wave power at higher densities without current drive. Observations of large Langmuir probe saturation currents due to lower hybrid wave power at high density were attributed to thermoelectric currents in the SOL plasma. ${ }^{11}$ Measurable parametric decay instabilities in the edge coincide with the onset of the current drive efficiency loss. ${ }^{19}$ Changes in H-mode quality, in the pedestal, and in the edge fluctuations were observed due to LH wave power in regimes with negligible current drive. ${ }^{20,21}$ Several separate observed phenomena highlight the importance of the LH wave power in the edge plasma at high density.

This work determines the deposition of lower hybrid wave power in the edge across the density range of current drive loss. This work finds the LH wave power is increasingly deposited outside the core plasma and near or outside the separatrix at higher densities. The power balance of the plasma was determined using a set of steady plasmas with an applied radiofrequency power modulation. Through the isolation of the applied power, it was possible to make several conclusions about the loss of current drive efficiency at densities greater than $\sim 1 \times 10^{20}\left(\mathrm{~m}^{-3}\right)$. Lower hybrid wave power loss shows a high degree of symmetry with the power absorbed near the separatrix. This power ionizes neutrals in the active divertor and is affected by edge recycling. These results suggest that prolonged propagation of lower hybrid waves through collisional regions outside the core of the plasma must be avoided in order to maintain good currentdrive efficiency.

The paper is organized as follows: the power balance of modulated LH wave power is examined. In Section II, the modulation technique is discussed and the effect on the core bulk plasma is analyzed. Minimal variation in the core was observed without current drive, allowing for changes in stored energy to be neglected at high density. Lack of core absorption was balanced with edge deposition. In Section III, the fast response of the edge plasma is used to determine the local deposition LH power and it is found that the fraction of power absorbed in the edge increases with density. However, the power lost in the edge was found to be increasingly radiated rather than conducted when the onset of high-recycling conditions in the divertor. In Section IV, the lower hybrid power is shown to ionize neutrals in the divertor region. The radiation and conduction of lower hybrid power in the edge can be used to localize the edge absorption. In Section V, it is found that the edge deposition occurs with a high degree of toroidal symmetry near the separatrix. The character of the divertor is dependent on the $\vec{B} \times \nabla|\vec{B}|$ direction, and in Section VI its effect on the symmetries of edge power absorption is determined. Finally, Section VII discusses how the previous results are consistent with the collisionality of the divertor plasma playing a key role. This work suggests that the propagation of lower hybrid waves through highly collisional plasma must be minimized if efficient current drive in the core is desired.

\section{LH MODULATION TECHNIQUES AND CORE POWER DEPOSITION}

The analysis of auxiliary power deposition in tokamaks requires isolating its effects from the background plasma. One such method for isolation is through the characterization of a step response of the plasma to input power. Using several such steps between two power levels (i.e., modulating the power), the accuracy and precision of the analysis can be improved. The subtle variation in background plasma parameters can be removed while improving the statistical rigor through multiple modulations. ${ }^{17,22,23}$ Power modulation is used in this paper to analyze the deposition of lower hybrid waves across the density range where current drive is lost.

The modulation of auxiliary power has been used extensively for the deposition of radiofrequency waves in tokamak plasmas. ${ }^{17,24-28}$ However, stringent requirements exist for the chosen power modulation. The modulated power must be a non-negligible fraction of the total power to allow for a significant plasma response to be determined. The timescale of the modulation must be long enough to fully capture the plasma dynamics. Previous work on core-deposited power required modulation timescales to be dictated by heat diffusive and convective transport of the thermal core plasma. The observation of deposited modulated power requires different measurement and analysis depending on the location (thermal or fast) and population (ion or electron).

Fast electron dynamics on C-Mod and other tokamaks have been characterized using LHCD power modulation. ${ }^{17,27,28}$ Measurements of high energy bremsstrahlung were used to isolate the response of the fast electron population due to LHCD. The timescales of fast electron analysis rely on the slowing down time, convective, and diffusive times which can be drastically different from the plasma thermal properties. In the work presented here, the modulation technique was not used to understand the fast electron dynamics, since there are relatively few fast electrons at the higher densities. Instead modulation is used to understand the LHCD effects on other core and edge plasma populations. The chosen modulation time $(0.1 \mathrm{~s}, 50 \%$ duty cycle) is longer than the energy confinement time $\left(\tau_{E} \sim 35 \mathrm{~ms}\right)$, allowing for the thermal distributions to be analyzed in the core. The rise/fall time between two modulated power levels is quite fast (less than $10 \mu \mathrm{s}$ ), allowing for the change in power to be effectively instantaneous.

Radial profiles of the bulk electron temperature were measured using a multiple point Thomson scattering system. ${ }^{29}$ Unlike measurements of electron cyclotron emission, these measurements are minimally affected by non-thermal electrons, allowing for the bulk electron dynamics to be measured. Five modulations within a single discharge were ensemble averaged at six different phases during the modulation. At low density, LHCD power replaces ohmic power 
causing the overall input power to be relatively constant. However, the lower efficiency of LHCD at higher density leads to minimal variation in the ohmic power. In the case shown in Figure 1, the input lower hybrid power represents a significant change in the total power into the vessel.

Electron temperature data from a diverted discharge without current drive were analyzed using an advanced profile fitting routine. ${ }^{30}$ These profiles were found to be self-similar within the variance in temperature across the radius of measurement. The lack of variation between profiles with and without Lower Hybrid Radiofrequency (LHRF) power suggests that there is little to no transfer of power of LH waves to the bulk electrons in cases without current drive. The ion population was also found to not respond to LH wave power as weak variation in neutron rate, which can be attributed to changes in core density, was caused by modulation pulses. A small change in the line-average density was observed in response to the modulated power, which is discussed in Section IV. Invariance of the core ion and electron temperatures with applied LHRF power shows the absence of core absorption.

In cases without current drive, the lack of variation in the bulk populations correlates with a lack of a fast electron population. This observation allows for the simplification of power balance at high density by assuming the stored energy of the plasma is constant with or without applied LHRF power. Without absorption of LHRF power in the plasma for $\rho<0.95$, the changes in input power are limited to the plasma near the periphery and will be affected by loss mechanisms existing there. In both cases, the response is dictated by the weak confinement of the edge plasma and should occur nearly instantaneously as radiation or conduction in the SOL.

By the process of elimination, it is now evident that the total deposition of LH wave power occurs in the edge for plasmas without current drive. However, it is the transition from current drive and the subsequent change in deposition profile that is more telling regarding the nature of the current

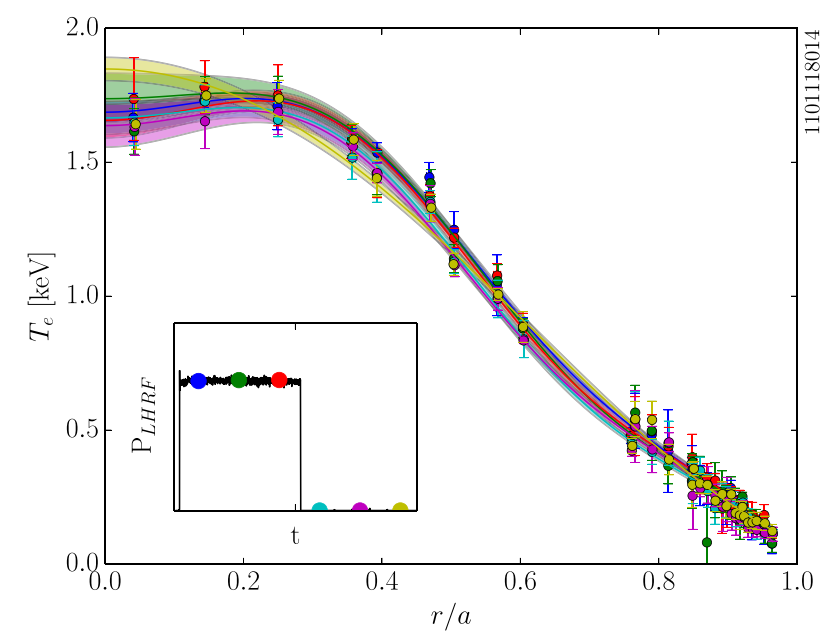

FIG. 1. Ensemble-averaged Thomson scattering $T_{e}$ profiles from six separate times (shown as inset) show minimal variation throughout the modulation at high density $\left(\bar{n}_{e}=1.1 \times 10^{20}\left(\mathrm{~m}^{-3}\right)\right)$. Single $\sigma$ error bars of the MAP estimate shown in color, with differences in the profiles within that range. This suggests that high density deposition of LHRF does not occur in the core plasma. drive loss. The observation of alternate absorption mechanisms in cases with partial current drive can identify the transition to edge power absorption and loss at the higher densities. Section III will discuss the power balance as it pertains to edge absorption with the loss of current drive.

\section{OBSERVATION OF LH POWER IN THE EDGE AND SOL}

\section{A. Experimental methodology}

The loss of current drive occurs over a range of density and has been found to be dependent on the plasma current. At higher currents, the loss in LHCD is less pronounced, increasing the range at which LHCD can be effectively used. ${ }^{31}$ This change in the current drive loss is accompanied by significant changes in the SOL. SOL widths (for the same $\overline{n_{e}}$ ) decrease, ${ }^{32}$ and detachment thresholds increase with higher plasma currents. The variability of the SOL due to parameters like the plasma current requires that independent variables be well controlled in these experiments.

The covariance of current drive loss with other parameters was minimized through the use of a constant shape, magnetic field, and plasma current. The transition region of density was probed using modulations in a single plasma topology at several steady densities. The set of plasma discharges with a lower single null equilibrium, 5.4T toroidal magnetic field, and a plasma current of $700 \mathrm{kA}$ were found to have a transition density range of $\bar{n}_{e}=7 \times 10^{19}$ to $1.3 \times 10^{20}\left(\mathrm{~m}^{-3}\right)$. Each modulation was treated separately, creating a database for quantifying observed edge and SOL power loss mechanisms.

The modulated lower hybrid wave power was $\sim 400 \mathrm{~kW}$, with an ohmic background power of $700 \mathrm{~kW}$. The modulation represents a $50 \%$ increase in the total power into the vessel when ohmic power remains constant. However, because the plasma current is under feedback control, the LH power replaces ohmic power (by reducing the loop voltage) within the plasma when, at lower densities, the LH wave can drive current. As the density rises, the current drive efficiency falls thereby diminishing the effect on the ohmic power. This causes the total power into the vessel to increase with higher density. The density dependence of the injected power is balanced by increased observed losses via conduction and radiation.

Two measures of SOL power loss are shown at three separate densities in Figure 2. The conducted power is observed via direct measurements of the outer strike point heat flux using fast thermocouples. ${ }^{33}$ Measurements of the active divertor $\mathrm{Ly}_{\alpha}$ emission are local to the SOL and are indicative of the edge radiative loss. Across the density range of current drive loss, the time response to lower hybrid wave power in the SOL is nearly instantaneous (with a response time of $<1 \mathrm{~ms}$ ). The prompt response of the SOL to the modulation increases in magnitude with increasing density.

The fast response $\left(t<\tau_{E}\right)$ of the edge to LH wave power simplifies the calculations of edge deposition. Rather than rely on Fourier techniques for analysis, the immediate response reduces the calculation of edge power loss to a subtraction. The deposited LH wave power in the edge 

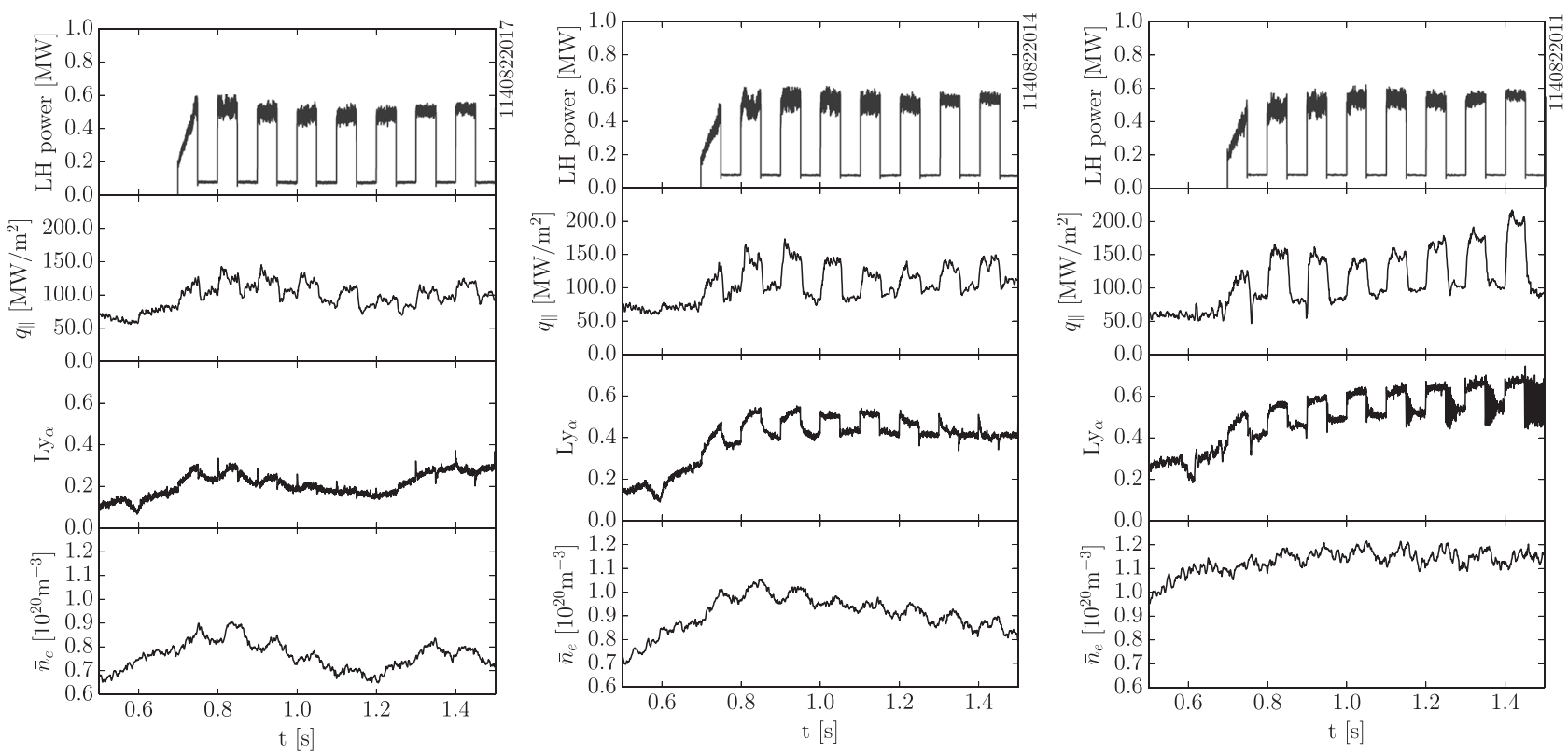

FIG. 2. Three plasmas at different densities. The response of the divertor plasma to lower hybrid wave power is observed in conduction $\left(q_{\|}\right.$and radiation $\left(\mathrm{Ly}_{\alpha}\right)$. The magnitude increases with density, while the time response is near instantaneous. The $\mathrm{Ly}_{\alpha}$ measurement is from the lower divertor in a lower single null plasma. The rise in heat flux and radiation coming from the additional power of lower hybrid waves which is no longer replacing ohmic power (e.g., $P_{t o t}$ is a function of density). Fast oscillations in the $\mathrm{Ly}_{\alpha}$ observed in the highest density case due to oscillations in the divertor neutral pressure.

$\left(\Delta P_{\text {LH.edge }}\right)$ can be calculated by the instantaneous change in the radiated power $\left(P_{\text {rad }}\right)$ and in the conducted power $\left(P_{\text {cond }}\right)$

$$
\Delta P_{\text {LH,edge }}=\Delta P_{\text {rad }}+\Delta P_{\text {cond }} .
$$

Dividing Equation (1) by the modulated net radiofrequency power $\Delta P_{L H}$, the fraction of input power absorbed in the edge can be calculated. While variation may occur in the core (e.g., small changes in the stored energy or replacement of the ohmic power by LHCD), the instantaneous response separates the direct edge deposition. The quantification of edge absorption of lower hybrid waves relies on the summation of instantaneous changes in the radiated and conducted power fractions.

\section{B. Edge loss conducted power}

The power conducted through the SOL in diverted discharges is predominantly observed on the inner and outer divertor targets. Assuming toroidal axisymmetry, these heat flux profiles on the targets are used to calculate the total integrated power on to the divertor plates. The measured $\mathrm{LH}$ wave power in the edge through conduction is treated as a combination of the prompt inner and outer instantaneous deposition. The geometrical separation of the inner and outer divertor requires characterizing each conductive loss independently.

The heat flux profiles on the inner and outer divertor are derived using infrared (IR) thermography ${ }^{34}$ and Langmuir probe arrays. Multiple measurements of the heat flux display the prompt nature of the edge LH wave power deposition on the outer divertor. Each measurement is assumed to axisymmetric, which is integrated toroidally for the total power. The observed rise in the conducted power occurs at or faster than the sampling time of these diagnostics $(t \sim 1 \mathrm{~ms})$. As shown in Figure 3, the conducted power as measured by IR thermography and Langmuir probes contains a clear rise in conducted power due to LHRF. Using subtraction, the conducted power was observed to be up to half of the input lower hybrid power in cases with minimal current drive.

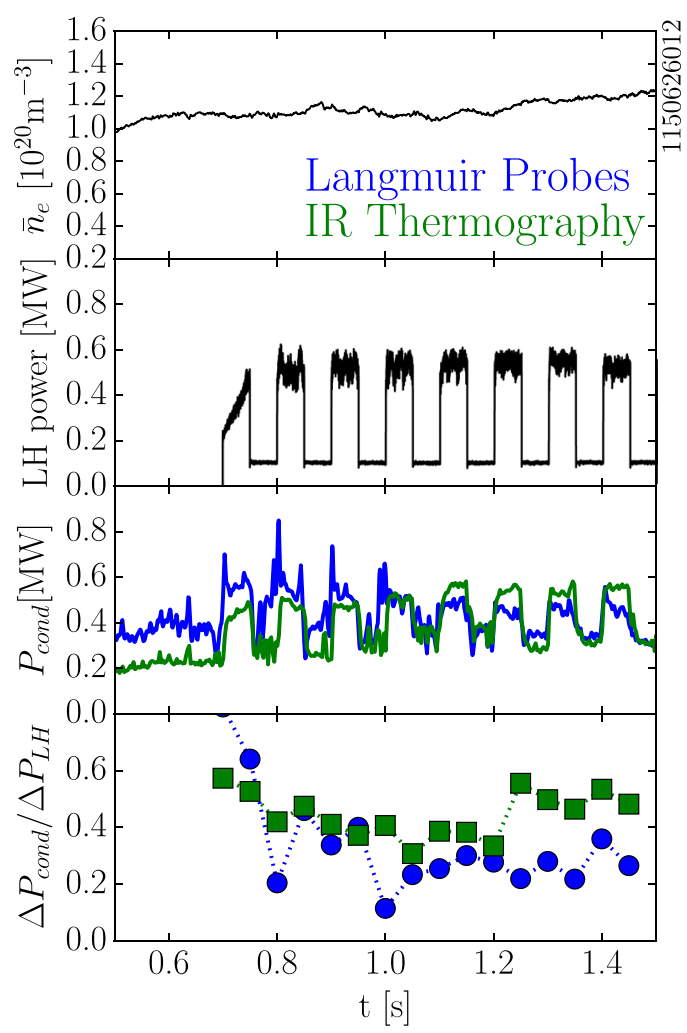

FIG. 3. Measurement of the total heat conducted to the outer divertor (with the outer leg is attached). A significant amount of the lower hybrid wave power conducted to the divertor, in the order of $40 \%$. Langmuir probe calculations assume a sheath heat flux transmission coefficient $(\gamma)$ of 7 . Calculation assumes toroidal axisymmetry. 
In forward field, measurements of the inner divertor show minimal change in the conducted power. The measured conduction to the inner divertor target never exceeds $12 \%$ of the input lower hybrid wave power. This is likely due to detachment of the inner divertor, which occurs at a lower line-average density near the onset of efficiency loss. Across the density range used in the experiment, observed power loss in the inner divertor region was predominantly radiative. Understanding the loss of power via radiation is a key for determining the fraction of lower hybrid power which is absorbed in the edge.

\section{Edge loss radiated power and power balance}

Measurement of the total radiated power was made using a foil bolometer. ${ }^{35}$ The wide view measures most of the poloidal cross-section except for a portion of the outer divertor leg and strike point. The radiated power from this measurement was characterized across the density range of current drive loss. Changes in $\Delta P_{\text {rad }}$ did not occur at low densities with significant current drive. However, the fraction of lower hybrid wave power lost via edge radiation increases with density. A fitted trend in the edge radiated fraction displays an increase from $\sim 0 \%$ to $>30 \%$ across the density range.

The edge radiated fraction, shown in Figure 4, highlights the increase in edge radiative loss with increasing density. Changes in the divertor associated with higher operational densities affect the channels of observed power loss. Higher density plasmas are more likely to have SOL plasmas in the high recycling regime, which leads to edge deposited lower hybrid power to be increasingly radiated. While the total edge deposition fraction still remains significant, the significance of some edge losses changes. At extremely high densities, changes to the SOL (i.e., detachment) will lead to the relative fraction of edge power lost via conduction and radiation to change, with most edge loss power being observed as radiation.

The fast time response of conduction and edge radiation increases in magnitude with higher densities. This increased deposition of lower hybrid wave power in the edge is

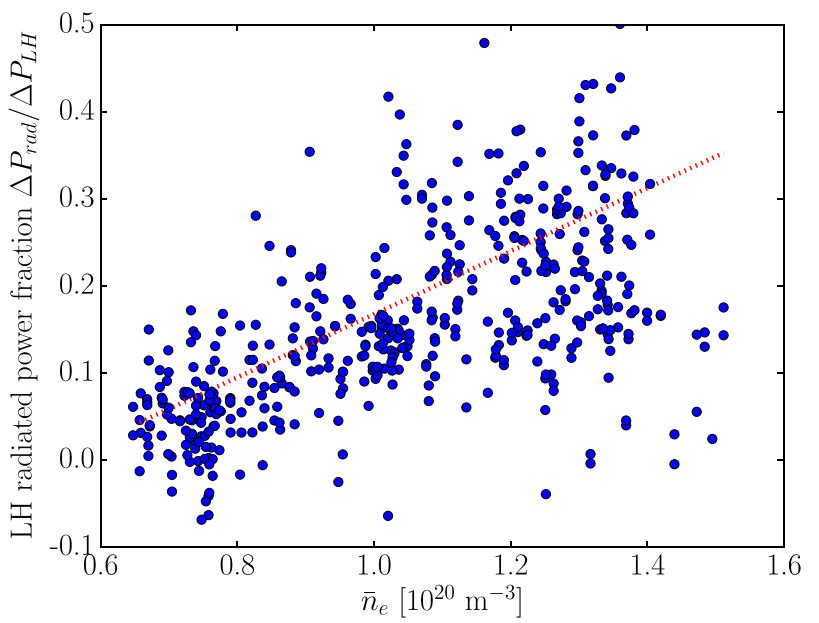

FIG. 4. Fraction of lower hybrid wave power lost as radiation. Data shown are in "forward field." The radiated loss starts near zero and rises across the range of current drive loss. Fitted trend line shows nearly 30\% of lower hybrid power is radiated at high density (with minimal current drive). matched with decreasing LHCD efficiency. The similar time response but changing magnitude reflects that the loss of efficiency does not come from a change in location of core deposition. Determination of the edge deposition confirms that the power loss is a parasitic mechanism near the edge which becomes stronger with density. The magnitude of power directly absorbed in the edge is strongly dependent on density, which accounts for the sudden decrease in current drive.

Shown in Table I is an example power balance at $\bar{n}_{e}=$ $1.2 \times 10^{20}\left(\mathrm{~m}^{-3}\right)$. Roughly $80 \%$ of the lower hybrid net power is observed to be promptly deposited in the edge plasma. A small fraction can still drive current, changing the loop voltage less than $10 \%$. The distribution of power loss in the value and channel is similar to the ohmic background and is specific to this density and plasma current. With the observed power balance, characteristics of edge power loss can now be determined.

\section{IONIZATION OF THE ACTIVE DIVERTOR}

Ionization and recombination in the plasma were measured using a polodially viewing 22 chord, AXUV diodebased, pinhole camera which measures the $\mathrm{Ly}_{\alpha}$ transition. The 2 to 1 transition of hydrogen and deuterium was isolated with a $\mathrm{MgF}_{2}$ filter, and the instrument was designed to characterize the variation in the SOL neutral/plasma balance under the influence of edge deposited lower hybrid wave power. Previous tangentially viewing observations found shifts in the emissivity profiles of $\mathrm{Ly}_{\alpha}$ and $\mathrm{He}-\mathrm{I}$ for deuterium and helium discharges, respectively ${ }^{36}$ (i.e., profiles moved outward in $\rho$ ). This diagnostic measures across most of the plasma cross section at a single toroidal location that is offset from the $\mathrm{LH}$ launcher by $36^{\circ}$ toroidally. It can discern changes in ionization and recombination local to the main plasma and inner divertor in almost all plasma topologies.

Figure 5 shows three plasmas with different divertor configurations at high density with applied lower hybrid wave power. The immediate addition of lower hybrid wave power is exhibited in the active divertor in all cases at high density. The instantaneous rise in the $\mathrm{Ly}_{\alpha}$ emission signifies that the divertor plasma changes due to the additional power in the edge. The neutral pressure in the divertor, shown in Figure 6, drops with applied radiofrequency power. The changes in $\mathrm{Ly}_{\alpha}$ and the neutral pressure appear to be caused

TABLE I. Observed power balance of discharge with significant edge absorption of lower hybrid power $\left(\bar{n}_{e}=1.15 \times 10^{20}\left(\mathrm{~m}^{-3}\right), B_{T}=5.4 \mathrm{~T}\right.$, $I_{p}=700 \mathrm{kA}$, forward field). The isolated net lower hybrid power generates a small fraction of the total current at this density, with edge absorbed power resembling the ohmic power loss. Errors on each measurement are of the order of $\sim 4 \%$ for the Ohmic power balance per measurement, and $\sim 6 \%$ for the lower hybrid power balance.

\begin{tabular}{lcc}
\hline \hline Loss & Ohmic $\%$ & LHRF $\%$ \\
\hline$\Delta P_{\text {OH }}$ & $\ldots$ & 18 \\
$P_{\text {cond,outer }}$ & 43 & 47 \\
$P_{\text {cond,inner }}$ & 7 & 6 \\
$P_{\text {rad,outer }}$ & 10 & 6 \\
$P_{\text {rad,inner }}$ & 25 & 17 \\
$P_{\text {rad,core }}$ & 15 & 6 \\
\hline \hline
\end{tabular}



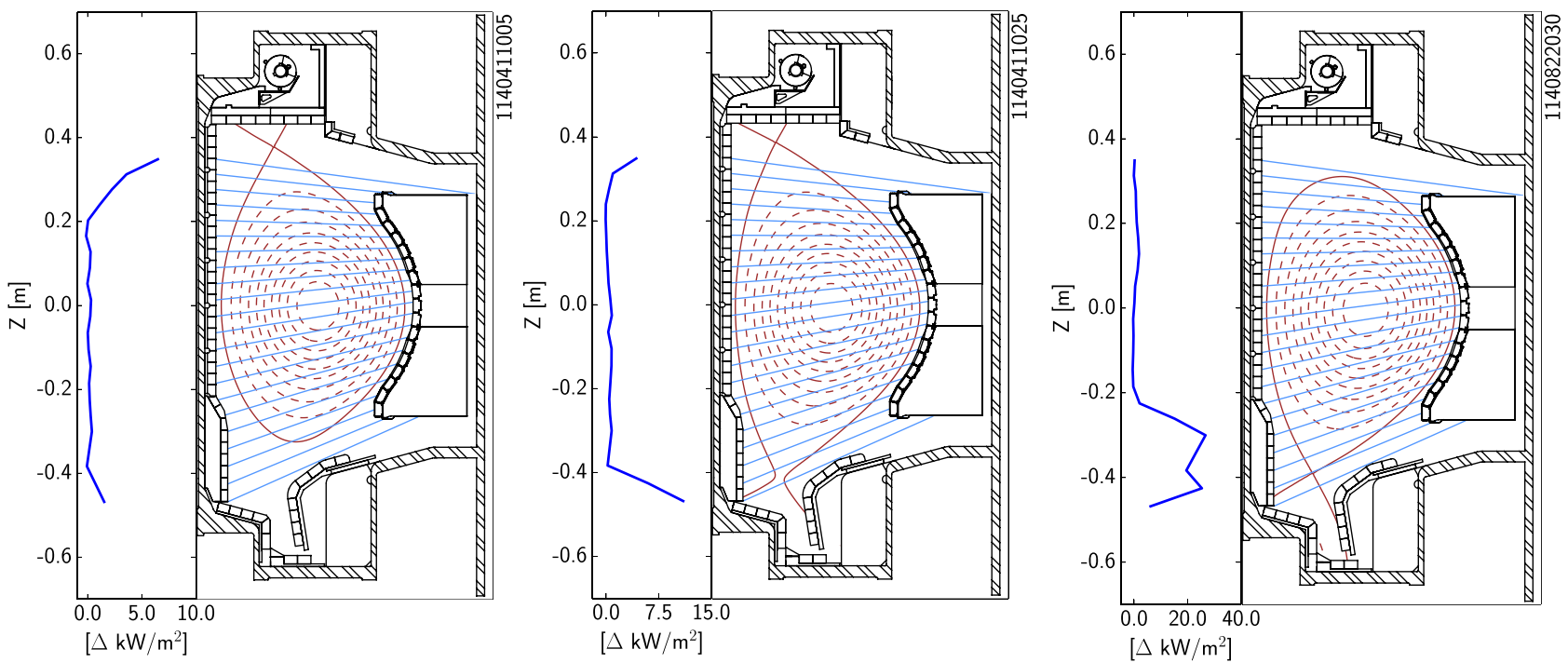

FIG. 5. Three plasmas at different densities. The response of the divertor plasma to lower hybrid wave power is observed in conduction and radiation. The magnitude increases with density, while the time response is near instantaneous. The Ly $\mathrm{L}_{\alpha}$ measurement is of the lower divertor in a lower single null plasma.

by the edge-deposited power ionizing neutrals in the active divertor.

The ionization in the divertor has a feedback effect on the core density. At particle confinement timescales, the line-average density rises in phase with the modulated power. This effect becomes more pronounced with higher plasma densities and is proportional to the applied power where minimal current drive is observed. In some cases, transitions to $\mathrm{H}$-mode due to the lower hybrid wave power were observed in the forward field. A $50 \%$ change in the injected power caused a $\sim 10 \%$ change in the $\bar{n}_{e}$.

The density of the inner divertor, as measured by the divertor Langmuir probes, rises with applied power due to

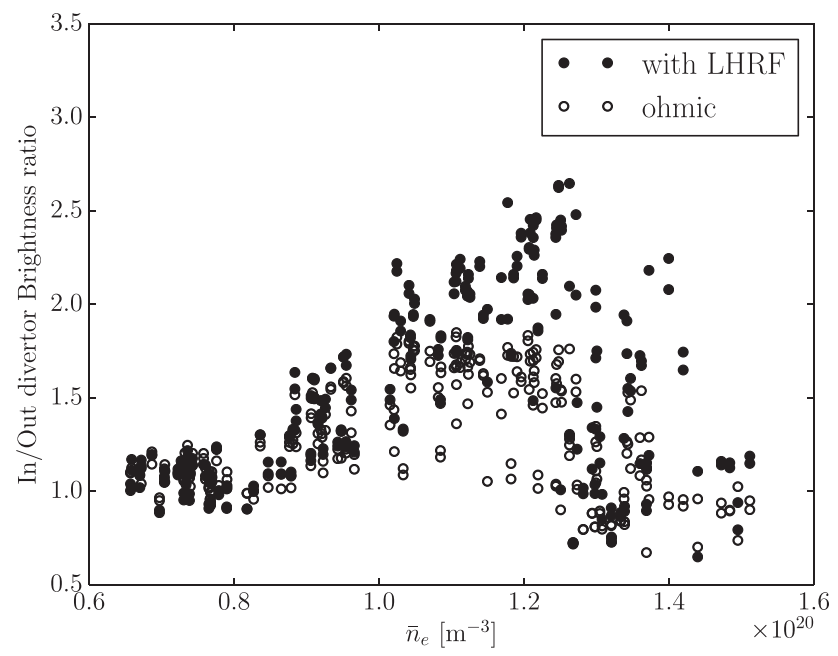

FIG. 6. The observed in/out asymmetry of radiative emission is enhanced by applied lower hybrid wave power at high density. The asymmetry in forward field lower single null discharges is due to the detachment of the inner divertor at a lower density than the outer divertor (shown as open circles). The ratio is higher with applied LHRF (shown with solid circles) due to enhanced emission from the inner divertor leg. The emission becomes more symmetrical as the outer leg detaches, with densities near $1.25 \times 10^{20}\left(\mathrm{~m}^{-3}\right)$. ionization. Minimal changes in the electron temperature were observed at the inner divertor target. This result is likely due to the very cold nature of the inner divertor leg in high density plasmas, nearing the threshold for detachment. The creation of density on the inner leg and related onset of detachment of the inner leg should show differences in the emission of radiation between in the inner and outer leg.

Measurements of the divertor radiated power with an AXUV diode array pinhole camera show significant enhancement of radiation from the inner leg. The ratio of emission from the inner to outer divertor legs is shown in Figure 6 (using two chord brightnesses). In purely ohmic plasmas, the ratio is balanced at low density and becomes weighted to the inner leg with inner leg detachment. Application of lower hybrid power enhances this in/out divertor asymmetry. This signifies that most of the measured change in $\mathrm{Ly}_{\alpha}$ and radiation is occurring on the inboard side of the divertor. The balance returns as the outer divertor plasma transitions to the high-recycling regime.

The edge deposited lower hybrid wave power dramatically changes the conditions of the active divertor. The changes in ionization observed in all plasma shapes focus the flow of power from the edge loss mechanism to this region. Localization of this power near the divertor can help to understand further the mechanism that reduces the current drive efficiency.

\section{RADIAL AND TOROIDAL LOCALIZATION OF DEPOSITION}

The macroscopic trends in edge power loss and the importance of the active divertor have shown the lower hybrid wave power absorption to occur in the edge. Additional measurements of prompt power in radiation and conduction from many locations can radially and toroidally localize the deposition of power in the edge. 
The radiated emission was measured using four toroidally separated diodes that view a narrow toroidal slice of the core plasma, $x$-point, and inner divertor. ${ }^{37}$ As shown in Figure 7, the response to the modulation is the same for each measurement. This suggests that the instantaneous power loss has a high degree of toroidal symmetry. In several cases, toroidally separated measures of conducted power via Langmuir probes were also found to be similar. Wide-angle views of the divertor using visible cameras show a large increase in light emission from the active divertor. The previously assumed axisymmetry of deposition assumed for edge power quantification is validated by this result.

The toroidally axisymmetric deposition of lower hybrid waves contrasts with other documented circumstances of edge deposition of radiofrequency waves. ${ }^{38,39}$ In these cases, the absorption has been found to be highly asymmetric with significant radiative emission measured along field lines connected to the antenna. The symmetry of emission observed in the active divertor suggests that other local measurements of the heat flux likely represent the axisymmetric character (such as the heat flux profiles). Therefore, the loss characteristics in the edge are not limited to regions near the launcher, and we conclude that the changes to the lower hybrid waves are unlikely to occur at that location only.

Figure 8 shows the parallel heat flux profiles on the outer divertor target mapped to the midplane and plotted versus $r_{\text {mid }}-r_{L C F S}$ using two separate measurements. In the cases where the divertor targets are attached, the instantaneous addition of power is predominantly observed near the strike point. A smaller increase in the heat flux is observed in the far SOL. The decay length of the heat flux in the near-SOL is similar with and without lower hybrid wave power. This implies that the deposition of power is occurring in the edge near the last closed flux surface.

The toroidally axisymmetric deposition of lower hybrid wave power near the last closed flux surface leads to two possible conclusions. One is that absorption of

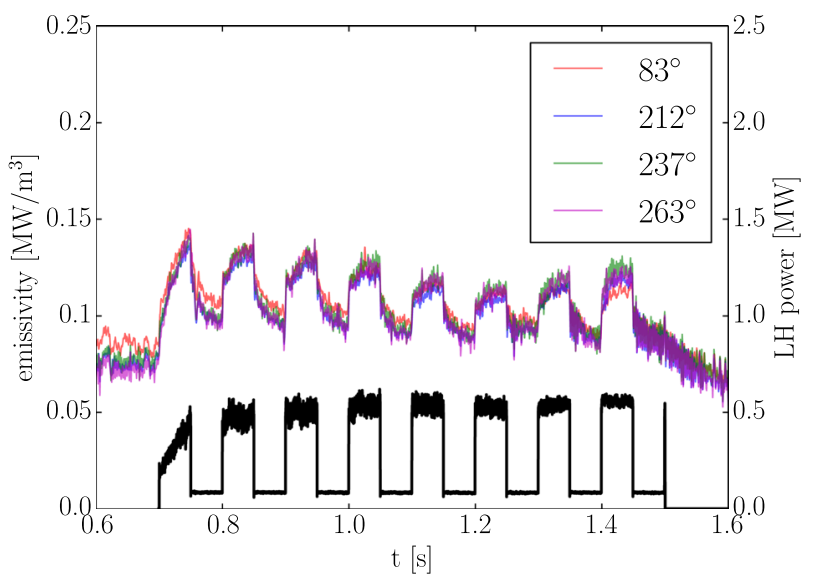

FIG. 7. Measurement of radiated power during modulation at high density shown at four locations offset from LHRF launcher (defined by toroidal angle in degrees) at high density. All signals respond similarly, suggesting that the prompt radiation emission from the edge is highly toroidally symmetric. At low density, the rise time in all the signals follows core confinement time, suggesting changes in the core temperature. The view is a wideangle poloidal view with a narrow toroidal slice representing the polodial dynamics at a specific toroidal location.

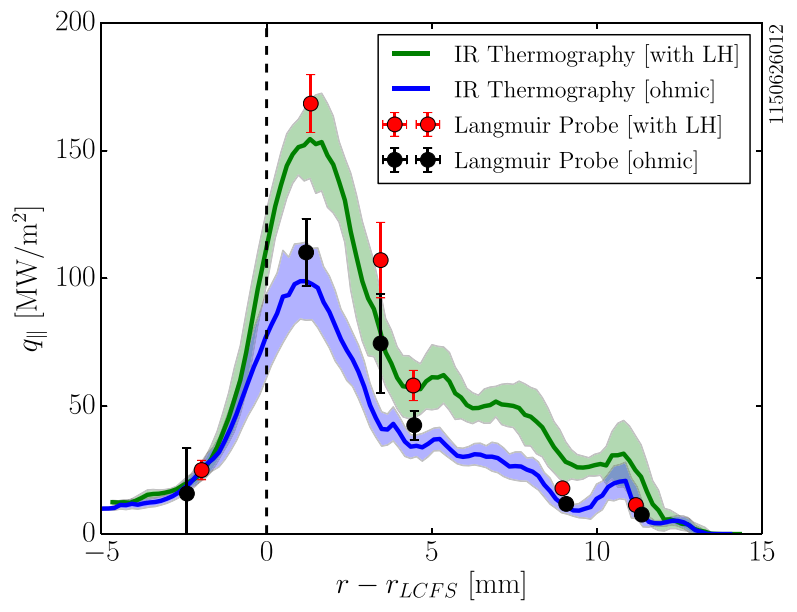

FIG. 8. Heat flux profiles on the attached outer divertor surface as measured by Langmuir Probes (assuming $\gamma=7$ ) and IR thermography during the two phases of modulation. The rise in measured parallel heat flux due to lower hybrid wave power is concentrated near the strike point (shown by the dashed line). The location of the lower hybrid wave edge deposition is correlated with the most collisional plasma near the strike point.

radiofrequency power occurs just inside the separatrix and quickly diffuses into the SOL. The other possibility is the absorption occurs just outside the separatrix, where the high density and low temperature provide the conditions for a strong collisional absorption. The waves symmetrize with the many traversals through the SOL, but preferentially lose their power near the LCFS leading to symmetrized power loss. Varying the conditions of the divertor can help shed light on which of these possibilities are more likely.

\section{REVERSED FIELD EFFECTS}

The characteristics of the divertor SOL change with the $\vec{B} \times \nabla|\vec{B}|$ direction. $^{40-42}$ The previous characteristics and trends were derived in so-called "forward field," where the $\vec{B} \times \nabla|\vec{B}|$ direction points toward the lower divertor. The inner divertor plasma in the forward field lower single null is cooler and denser than the outer divertor. This leads to detachment of the inner divertor at lower line-average densities than the outer divertor. The inner divertor conducts significantly less power than the outer divertor. These attributes were observed for most of the range of interest both with the edge deposited lower hybrid power and the power of the background plasma.

The current and magnetic field are in the opposite direction in the "reversed-field" configuration on C-Mod, leading to the $\vec{B} \times \nabla|\vec{B}|$ direction pointing upwards toward the upper divertor. In this condition, the divertor heat flux in lower single null is roughly balanced between the inner and outer divertor. The inner divertor is hotter and less dense compared to the forward-field configuration for the same average density, leading to a lower collisionality. The inner and outer divertor plasmas detach at similar densities due to the comparable densities and temperatures. The change in divertor conditions provided by such a change in field-direction can be used to test conclusions regarding the edge deposition of lower hybrid waves. 
The experimental conditions of the forward field plasmas were replicated in reversed field across the same range in density. In Figure 9, two plasmas with the same lineaverage densities are shown in the forward and reverse field. While the modulation response is observed strongly in the $\mathrm{Ly}_{\alpha}$ emission in the divertor in the forward field, it is absent in reversed field. This difference is similarly observed in the total radiated power. Significant differences in the radiation induced by edge deposited lower hybrid waves are observed with changes in field direction.

As shown in Figure 10, the fraction of lower hybrid wave power radiated in the edge is near zero for densities below $1.1 \times 10^{20}\left(\mathrm{~m}^{-3}\right)$. Above this density, the observed radiated power loss rises to at most $20 \%$ of the input lower hybrid power. The onset of edge power loss via radiation at higher density correlates with the less collisional nature of reversed-field divertor plasmas. The radiation of edge deposited lower hybrid power mimics changes in SOL radiation associated with field reversal.

Measurements of the conducted power due to edge deposited lower hybrid waves also show similarities to general conducted power in reversed field. Langmuir probe arrays on the inner and outer divertors yield similar instantaneous conducted powers in plasmas without detachment. This result is

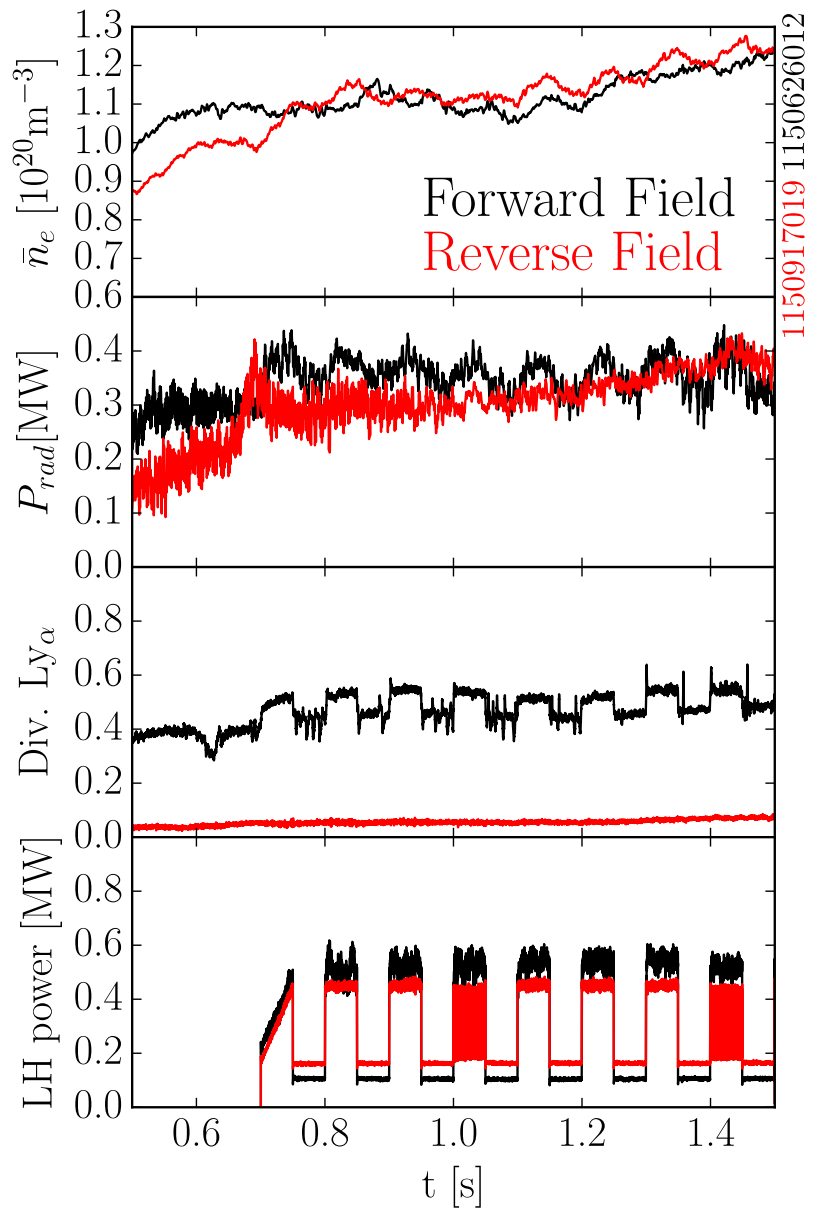

FIG. 9. Two discharges with similar shape, magnetic field, current and density are shown for the forward and reversed field. The divertor response to lower hybrid wave power changes with the field direction. The modulation in radiated power of the divertor is not observed in this case, with significantly less $\mathrm{Ly}_{\alpha}$ radiation from the active divertor.

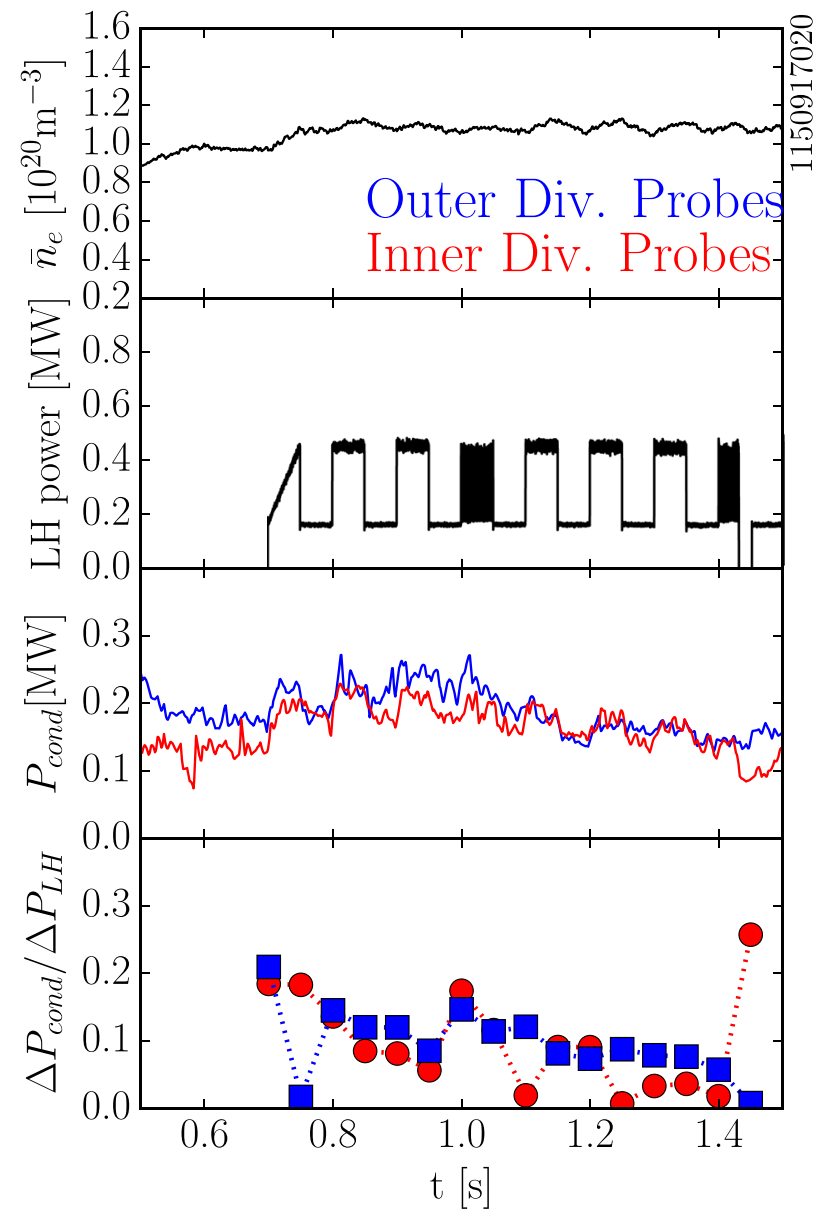

FIG. 10. The observed conducted power in reversed field on the inner and outer divertor are similar. Measurements were made using arrays of Langmuir probes on the inner and outer wall. The loss of power in the edge follows characteristic changes observed with the change in field.

shown in Figures 10 and 11 in a plasma with moderate density. The power loss characteristics of core thermal energy in the active divertor associated with field direction are also observed in edge deposited lower hybrid wave power. The

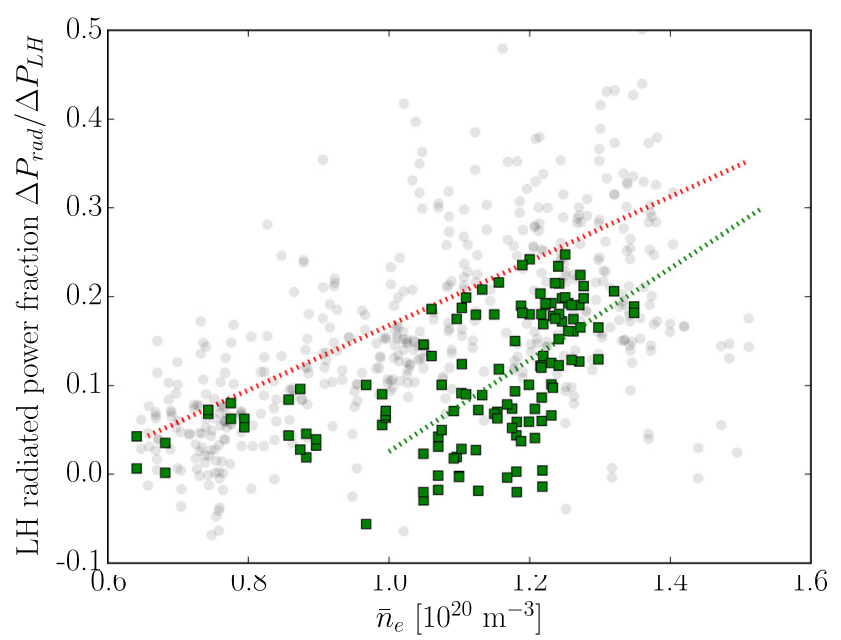

FIG. 11. Fraction of lower hybrid wave power lost as radiation. Data shown in green are "reverse field," with forward field data in gray. The radiated loss rises at a higher density than in forward field. This result follows changes in detachment and radiation observed with the change in field. 
mechanism causing lower hybrid wave power absorption in the edge must be correlated with SOL changes in the divertor, an observation that is highlighted by the reversed field results.

\section{DISCUSSION AND SUMMARY}

Power modulation techniques have been used to quantify the edge deposition of lower hybrid wave power. This work has confirmed that the edge plasma is the region responsible for the decreased current drive efficiency at high density. These observations have determined a number of characteristics about the loss of current drive and the transition to complete edge absorption. The mechanism behind the loss of current drive must be induced by the attributes of the divertor SOL plasma and has now been determined to contain several specific parasitic characteristics.

Mechanisms which modify the $n_{\|}$of the wave (such as PDI and fluctuation scattering) or frictionally absorb the wave in the plasma occur most strongly in regions of low temperature and high density (i.e., high collisionality). The existence of a cold, dense, and collisional divertor plasma correlates with the loss of current drive. The current and density dependence of current drive loss also correlates with similar changes of the SOL width and collisionality (which affect the size and intensity of the collisional zone). The observed ionization of the active divertor highlights the importance of this cold, dense region.

The region of highest collisionality, just outside the separatrix, displays the greatest change in heat flux from lower hybrid waves. The high degree of toroidal axisymmetry in deposition indicates the waves are making multiple traversals through the SOL. This process allows the waves to pass through this highly collisional region leading to absorption of the wave in the edge. Field direction can profoundly change the divertor plasma, which also changes the lower hybrid wave edge deposition. With the significant ionization and influence of recycling neutrals, these characteristics highlight the importance of the divertor plasma for edge deposition.

The importance of collisionality in the absorption of lower hybrid waves dictates that for good current-drive efficiency the propagating wave must avoid the divertor plasma. Future devices will require the use of a highly collisional detached zone for power dissipation in the divertor. This work highlights the importance of isolating the wave power from collisional regions. The propagation of lower hybrid rays must avoid highly collisional regions in order to efficiently drive current. These results motivate work in further understanding the propagation and deposition of LH waves for future high power tokamaks.

The core temperatures and expected wave-upshifts for ITER and DEMO-like LHCD systems will lead to first-pass damping of the waves in the core plasma and are unlikely to need multiple traversals through the SOL. While this contrasts the conditions in C-Mod, it is possible that future ITER or DEMO devices could have sufficiently cold and collisional scrape-off-layers leading to significant edge absorption. The long SOL propagation distances expected for radiofrequency systems in these devices $(>12 \mathrm{~cm}$ expected for ITER $^{43}$ ) could lead to significant absorption before entering the core plasma. It will be important to include accurate models of the SOL and associated absorption in designing LHRF systems for these devices.

However, the interaction of launched LH waves with collisional regions of plasma can be minimized. The avoidance of wave propagation through certain portions of the SOL can be made with a proper choice of launching location and launched $\mathrm{n}_{\|}$. The choice of a high field side launching location can improve core penetration of lower hybrid waves. When used in a double null plasma, high-field-side launch ${ }^{44}$ combines favorable SOL characteristics with efficient LH wave damping thereby minimizing edge absorption. ${ }^{45}$ Together, these methods can improve the efficacy of LHCD in next-step devices with high densities, high magnetic fields, and divertors.

As has been shown in this work on Alcator C-Mod, multiple passes of LH waves through core and edge lead to a significant interaction with the SOL plasma and can lead to launched waves being absorbed in the divertor plasma. The new conclusions derived from modulation highlight the complete parasitic loss of power in the edge. Plasmas with good SOL characteristics combined with effective wave damping in the core plasma can minimize the effect of collisional regions. Future LHCD systems for ITER and DEMO-like tokamaks must take into account the collisional nature of the edge plasma and associated absorption even in through the first traversal.

\section{ACKNOWLEDGMENTS}

This work was supported by the U.S. Department of Energy, Office of Science, Office of Fusion Energy Sciences, using User Facility Alcator C-Mod, under Award No. DEFC02-99ER54512-CMOD.

${ }^{1}$ J. Wilson, R. Parker, M. Bitter, P. Bonoli, C. Fiore, R. Harvey, K. Hill, A. Hubbard, J. Hughes, A. Ince-Cushman, C. Kessel, J. Ko, O. Meneghini, C. Phillips, M. Porkolab, J. Rice, A. Schmidt, S. Scott, S. Shiraiwa, E. Valeo, G. Wallace, J. Wright, and the Alcator C-Mod Team, "Lower hybrid heating and current drive on the Alcator C-Mod tokamak," Nucl. Fusion 49, 115015 (2009)

${ }^{2}$ N. J. Fisch, "Theory of current drive in plasmas," Rev. Mod. Phys. 59, 175-234 (1987).

${ }^{3}$ S. Shiraiwa, G. Baek, P. Bonoli, I. Faust, A. Hubbard, O. Meneghini, R. Parker, G. Wallace, J. Wilson, R. Harvey, A. Smirnov, D. Brunner, B. LaBombard, C. Lau, R. Mumgaard, S. Scott, N. Tsujii, S. Wolfe, and the Alcator C-Mod team, "Progress towards steady-state regimes in Alcator C-Mod," Nucl. Fusion 53, 113028 (2013).

${ }^{4}$ P. Bonoli, R. Parker, M. Porkolab, J. Ramos, S. Wukitch, Y. Takase, S. Bernabei, J. Hosea, G. Schilling, and J. Wilson, "Modelling of advanced tokamak scenarios with LHCD in Alcator C-Mod," Nucl. Fusion 40, 1251 (2000).

${ }^{5}$ A. Kaye and G. O'Connor, "High frequency heating and current drive in next step steady state tokamaks," Fusion Eng. Des. 56-57, 47-57 (2001).

${ }^{6}$ P. T. Bonoli and R. C. Englade, "Simulation model for lower hybrid current drive," Phys. Fluids 29, 2937-2950 (1986).

${ }^{7} \mathrm{M}$. Porkolab, "Theory of parametric instability near the lowerhybrid frequency," Phys. Fluids 17, 1432-1442 (1974).

${ }^{8}$ P. T. Bonoli and E. Ott, "Toroidal and scattering effects on lowerhybrid wave propagation," Phys. Fluids 25, 359-375 (1982).

${ }^{9}$ C. Castaldo, A. D. Siena, R. Fedele, F. Napoli, L. Amicucci, R. Cesario, and G. Schettini, "Influence of collisions on parametric instabilities 
induced by lower hybrid waves in tokamak plasmas," Nucl. Fusion 56, 016003 (2016).

${ }^{10}$ G. M. Wallace, R. R. Parker, P. T. Bonoli, A. E. Hubbard, J. W. Hughes, B. L. LaBombard, O. Meneghini, A. E. Schmidt, S. Shiraiwa, D. G. Whyte, J. C. Wright, S. J. Wukitch, R. W. Harvey, A. P. Smirnov, and J. R. Wilson, "Absorption of lower hybrid waves in the scrape off layer of a diverted tokamak,” Phys. Plasmas (1994-present) 17, 082508 (2010).

${ }^{11}$ G. Wallace, A. Hubbard, P. Bonoli, I. Faust, R. Harvey, J. Hughes, B. LaBombard, O. Meneghini, R. Parker, A. Schmidt, S. Shiraiwa, A. Smirnov, D. Whyte, J. Wilson, J. Wright, S. Wukitch, and the Alcator CMod Team, "Lower hybrid current drive at high density in Alcator CMod," Nucl. Fusion 51, 083032 (2011).

${ }^{12}$ J. Liptac, R. Parker, V. Tang, Y. Peysson, and J. Decker, "Hard x-ray diagnostic for lower hybrid experiments on Alcator C-Mod," Rev. Sci. Instrum. 77, 103504 (2006).

${ }^{13}$ M. Goniche, V. Basiuk, J. Decker, P. Sharma, G. Antar, G. Berger-By, F. Clairet, L. Delpech, A. Ekedahl, J. Gunn, J. Hillairet, X. Litaudon, D. Mazon, E. Nilsson, T. Oosako, Y. Peysson, M. Preynas, M. Prou, and J. Sgui, "Lower hybrid current drive at high density on tore supra," Nucl. Fusion 53, 033010 (2013).

${ }^{14}$ Y. Takase, M. Porkolab, J. J. Schuss, R. L. Watterson, C. L. Fiore, R. E. Slusher, and C. M. Surko, "Observation of parametric instabilities in the lowerhybrid range of frequencies in the highdensity tokamak," Phys. Fluids 28, 983-994 (1985).

${ }^{15}$ V. P. Ridolfini, M. Apicella, G. Calabr, C. Cianfarani, E. Giovannozzi, and L. Panaccione, "Lower hybrid current drive efficiency in tokamaks and wave scattering by density fluctuations at the plasma edge," Nucl. Fusion 51, 113023 (2011).

${ }^{16}$ K. Ushigusa, T. Kondoh, O. Naito, Y. Ikeda, S. Ide, M. Seki, S. Wolfe, M. Sato, Y. Kamada, K. Itami, and T. Imai, "Direct loss of energetic electrons during lower hybrid current drive in jt-60u," Nucl. Fusion 32, 1977 (1992).

${ }^{17}$ K. Kirov, Y. Baranov, J. Mailloux, M.-L. Mayoral, M. Nave, J. Ongena, and J. E. Contributors, "LH power deposition and CD efficiency studies by application of modulated power at JET," Nucl. Fusion 50, 075003 (2010).

${ }^{18}$ B. Ding, Y. Li, L. Zhang, M. Li, W. Wei, E. Kong, M. Wang, H. Xu, S. Wang, G. Xu, L. Zhao, H. Hu, H. Jia, M. Cheng, Y. Yang, L. Liu, H. Zhao, Y. Peysson, J. Decker, M. Goniche, L. Amicucci, R. Cesario, A. Tuccillo, S. Baek, R. Parker, P. Bonoli, F. Paoletti, C. Yang, J. Shan, F. Liu, Y. Zhao, X. Gong, L. Hu, X. Gao, B. Wan, J. Li, and the EAST team, "Investigations of lhw-plasma coupling and current drive at high density related to h-mode experiments in east," Nucl. Fusion 55, 093030 (2015).

${ }^{19}$ S. G. Baek, R. R. Parker, S. Shiraiwa, G. M. Wallace, P. T. Bonoli, D. Brunner, I. C. Faust, A. E. Hubbard, B. LaBombard, and M. Porkolab, "Measurements of ion cyclotron parametric decay of lower hybrid waves at the high-field side of alcator c-mod," Plasma Phys. Controlled Fusion 55, 052001 (2013).

${ }^{20}$ J. Hughes, A. Hubbard, G. Wallace, M. Greenwald, B. LaBombard, L. Lin, R. McDermott, R. Parker, M. Reinke, J. Rice, and J. Wilson, "Modification of H-mode pedestal structure with lower hybrid waves on Alcator C-Mod," Nucl. Fusion 50, 064001 (2010).

${ }^{21}$ J. L. Terry, M. L. Reinke, J. W. Hughes, B. LaBombard, C. Theiler, G. M. Wallace, S. G. Baek, D. Brunner, R. M. Churchill, E. Edlund, P. Ennever, I. Faust, T. Golfinopoulos, M. Greenwald, A. E. Hubbard, J. Irby, Y. Lin, R. R. Parker, J. E. Rice, S. Shiraiwa, J. R. Walk, S. J. Wukitch, P. Xu, and C.-M. Team, "Improved confinement in high-density H-modes via modification of the plasma boundary with lower hybrid waves," Phys. Plasmas 22, 056114 (2015).

${ }^{22}$ T. C. Luce, C. C. Petty, and J. C. M. de Haas, "Inward energy transport in tokamak plasmas," Phys. Rev. Lett. 68, 52-55 (1992).

${ }^{23}$ F. Ryter, R. Dux, P. Mantica, and T. Tala, "Perturbative studies of transport phenomena in fusion devices," Plasma Phys. Controlled Fusion 52, 124043 (2010)

${ }^{24} \mathrm{~V}$. Erckmann and U. Gasparino, "Electron cyclotron resonance heating and current drive in toroidal fusion plasmas," Plasma Phys. Controlled Fusion 36, 1869 (1994).

${ }^{25}$ D. Gambier, M. Evrard, J. Adam, A. Becoulet, S. Corti, P. Hennequin, J. Jacquinot, D. Start, K. Thomsen, B. Tubbing, and V. Zanza, "ICRF power deposition profile and determination of the electron thermal diffusivity by modulation experiments in JET," Nucl. Fusion 30, 23 (1990).

${ }^{26}$ T. Hellsten, M. Laxback, T. Bergkvist, T. Johnson, F. Meo, F. Nguyen, C. Petty, M. Mantsinen, G. Matthews, J.-M. Noterdaeme, T. Tala, D. V. Eester, P. Andrew, P. Beaumont, V. Bobkov, M. Brix, J. Brzozowski, L.G. Eriksson, C. Giroud, E. Joffrin, V. Kiptily, J. Mailloux, M.-L. Mayoral,
I. Monakhov, R. Sartori, A. Staebler, E. Rachlew, E. Tennfors, A. Tuccillo, A. Walden, K.-D. Zastrow, and J.-E. Contributors, "On the parasitic absorption in FWCD experiments in JET ITB plasmas,” Nucl. Fusion 45, 706 (2005).

${ }^{27}$ Y. Peysson, "Transport of fast electrons during LHCD in TS, JET, and ASDEX," Plasma Phys. Controlled Fusion 35, B253 (1993).

${ }^{28}$ A. Schmidt, P. T. Bonoli, O. Meneghini, R. R. Parker, M. Porkolab, S. Shiraiwa, G. Wallace, J. C. Wright, R. W. Harvey, and J. R. Wilson, "Investigation of lower hybrid physics through power modulation experiments on Alcator C-Mod," Phys. Plasmas (1994-present) 18, 056122 (2011).

${ }^{29}$ J. W. Hughes, D. Mossessian, K. Zhurovich, M. DeMaria, K. Jensen, and A. Hubbard, "Thomson scattering upgrades on Alcator C-Mod," Rev. Sci. Instrum. 74, 1667-1670 (2003).

${ }^{30}$ M. Chilenski, M. Greenwald, Y. Marzouk, N. Howard, A. White, J. Rice, and J. Walk, "Improved profile fitting and quantification of uncertainty in experimental measurements of impurity transport coefficients using Gaussian process regression," Nucl. Fusion 55, 023012 (2015).

${ }^{31}$ S. Baek, R. Parker, P. Bonoli, S. Shiraiwa, G. Wallace, B. LaBombard, I. Faust, M. Porkolab, and D. Whyte, "High density LHRF experiments in Alcator C-Mod and implications for reactor scale devices," Nucl. Fusion 55, 043009 (2015).

${ }^{32}$ T. Eich, B. Sieglin, A. Scarabosio, W. Fundamenski, R. J. Goldston, and A. Herrmann (ASDEX Upgrade Team), "Inter-ELM power decay length for JET and ASDEX Upgrade: Measurement and comparison with heuristic drift-based model," Phys. Rev. Lett. 107, 215001 (2011).

${ }^{33}$ D. Brunner and B. LaBombard, "Surface thermocouples for measurement of pulsed heat flux in the divertor of the Alcator C-Mod tokamak," Rev. Sci. Instrum. 83, 033501 (2012).

${ }^{34}$ J. L. Terry, B. LaBombard, D. Brunner, J. Payne, and G. A. Wurden, "Divertor IR thermography on Alcator C-Mod," Rev. Sci. Instrum. 81, 10E513 (2010).

${ }^{35}$ E. R. Mller and F. Mast, "A new metal resistor bolometer for measuring vacuum ultraviolet and soft x radiation," J. Appl. Phys. 55, 2635-2641 (1984).

${ }^{36}$ G. M. Wallace, I. C. Faust, O. Meneghini, R. R. Parker, S. Shiraiwa, S. G. Baek, P. T. Bonoli, A. E. Hubbard, J. W. Hughes, B. L. LaBombard, C. Lau, Y. Ma, M. L. Reinke, J. L. Terry, D. G. Whyte, J. C. Wright, S. J. Wukitch, R. W. Harvey, A. E. Schmidt, A. P. Smirnov, and J. R. Wilson, "Lower hybrid current drive at high density in the multi-pass regime," Phys. Plasmas 19, 062505 (2012).

${ }^{37}$ G. Olynyk, R. Granetz, M. Reinke, D. Whyte, T. Golfinopoulos, J. Hughes, J. Walk, V. Izzo, S. Combs, S. Milora, and M. Brookman, "Rapid shutdown experiments with one and two gas jets on alcator c-mod," Nucl. Fusion 53, 092001 (2013).

${ }^{38}$ Y. Liang, X. Z. Gong, K. F. Gan, E. Gauthier, L. Wang, M. Rack, Y. M. Wang, L. Zeng, P. Denner, A. Wingen, B. Lv, B. J. Ding, R. Chen, L. Q. Hu, J. S. Hu, F. K. Liu, Y. X. Jie, J. Pearson, J. P. Qian, J. F. Shan, B. Shen, T. H. Shi, Y. Sun, F. D. Wang, H. Q. Wang, M. Wang, Z. W. Wu, S. B. Zhang, T. Zhang, X. J. Zhang, N. Yan, G. S. Xu, H. Y. Guo, B. N. Wan, and J. G. Li, "Magnetic topology changes induced by lower hybrid waves and their profound effect on edge-localized modes in the east tokamak," Phys. Rev. Lett. 110, 235002 (2013).

${ }^{39}$ R. J. Perkins, J. C. Hosea, G. J. Kramer, J.-W. Ahn, R. E. Bell, A. Diallo, S. Gerhardt, T. K. Gray, D. L. Green, E. F. Jaeger, M. A. Jaworski, B. P. LeBlanc, A. McLean, R. Maingi, C. K. Phillips, L. Roquemore, P. M. Ryan, S. Sabbagh, G. Taylor, and J. R. Wilson, "High-harmonic fast-wave power flow along magnetic field lines in the scrape-off layer of nstx," Phys. Rev. Lett. 109, 045001 (2012).

${ }^{40}$ I. H. Hutchinson, B. LaBombard, J. A. Goetz, B. Lipschultz, G. M. McCracken, J. A. Snipes, and J. L. Terry, "The effects of field reversal on the alcator c-mod divertor," Plasma Phys. Controlled Fusion 37, 1389 (1995).

${ }^{41}$ W. Fundamenski, P. Andrew, K. Erents, A. Huber, G. Kirnev, G. Matthews, R. Pitts, V. Riccardo, and S. Sipil, "Effect of b b direction on $\{$ SOL $\}$ energy transport in $\{\mathrm{JET}\}, " J$. Nucl. Mater. 337-339, 305-309 (2005).

${ }^{42}$ S. C. Liu, H. Y. Guo, G. S. Xu, X. Gao, S. Z. Zhu, H. Q. Wang, L. Wang, N. Yan, D. S. Wang, P. Liu, M. Jiang, W. Zhang, T. F. Ming, J. F. Chang, S. Y. Ding, H. Xiong, L. M. Shao, Z. W. Wu, G. N. Luo, and E. Team, "Divertor asymmetry and scrape-off layer flow in various divertor configurations in experimental advanced superconducting tokamak," Phys. Plasmas 19, 042505 (2012). 
${ }^{43}$ A. Ekedahl, G. Granucci, J. Mailloux, Y. Baranov, S. Erents, E. Joffrin, X. Litaudon, A. Loarte, P. Lomas, D. McDonald, V. Petrzilka, K. Rantamki, F. Rimini, C. Silva, M. Stamp, A. Tuccillo, and J. E. Contributors, "Long distance coupling of lower hybrid waves in jet plasmas with edge and core transport barriers," Nucl. Fusion 45, 351 (2005).

${ }^{44}$ G. M. Wallace, S. G. Baek, P. T. Bonoli, I. C. Faust, B. L. LaBombard, Y. Lin, R. T. Mumgaard, R. R. Parker, S. Shiraiwa, R.
Vieira, D. G. Whyte, and S. J. Wukitch, "High field side launch of rf waves: A new approach to reactor actuators," AIP Conf. Proc. 1689, 030017 (2015).

${ }^{45}$ Y. Podpaly, G. Olynyk, M. Garrett, P. Bonoli, and D. Whyte, "The lower hybrid current drive system for steady-state operation of the vulcan tokamak conceptual design," Fusion Eng. Des. 87, 215-223 (2012), special Section on Vulcan Conceptual Design. 